\title{
DESENVOLVIMENTO E CARACTERIZAÇÃO DE FILMES DE COLÁGENO COM ADIÇÃO DE ÓLEO ESSENCIAL DE CRAVO-DA-ÍNDIA
}

\section{DEVELOPMENT AND CHARACTERIZATION OF COLLAGEN FILMS WITH ADDED ESSENTIAL OIL OF CLOVE INDIA}

\author{
Ailim Yuki NAKASHIMA ${ }^{[1]}$, Raquel Costa CHEVALIER ${ }^{[1]}$ e William Renzo CORTEZ-VEGA ${ }^{[1, *]}$ \\ [1] Universidade Federal da Grande Dourados, FAEN - Faculdade de Engenharia, Laboratório de Bioengenharia. Rod. Dourados km 12, Itahum. \\ 79804970 Dourados, MS - Brasil. email: ailimyuki@hotmail.com e quel.chevalier@hotmail.com
}

*Autor para correspondência: williamvega@ufgd.edu.br

\section{INFORMAÇÃO}

Recebido em: 10/12/2015

Aceito em: 23/03/2016

Publicado em: 28/03/2016

\section{Document Object Identifier}

$10.18067 / \mathrm{jbfs} . v 3 \mathrm{i} 1.86$

Editor: V. H. G. Sales

jbfs@ifap.edu.br

ID JBFS0862015

Avaliação a cega por pares

Prot. 0862016R01

Prot. 0862016RO2

Prot. 0862016R03

Copyright: (C) 2016

JBFS all rights (BY NC SA)

\section{RESUMO}

Este trabalho teve como objetivo desenvolver filmes à base de colágeno e analisar os efeitos das concentrações de argila, plastificante e óleo essencial sobre as características de cor, opacidade, resistência à tração, solubilidade, permeabilidade ao vapor da água e espessura dos filmes. Os atributos de cor são de grande importância na área de embalagens, pois influenciam diretamente na aceitabilidade do consumidor. Para o preparo de soluções filmogênicas, os níveis de fatores foram avaliados de acordo com um planejamento experimental de $2^{3}$ com 3 pontos centrais. Foram desenvolvidos pela técnica de "casting", que consiste na secagem em superfície. Os valores de luminosidade variaram entre 66,76 e 96,03 . Os valores de opacidade apresentaram aumento com a adição de glicerol, variando de 1,96 a 44,24\%. Os valores de resistência à tração variaram entre 5 a $14 \mathrm{MPa}$, e os valores solubilidade, de 2,14 a 7,97\%. Os resultados de solubilidade e de resistência à tração não foram significativos. Os valores de permeabilidade ao vapor de água (PVA) variaram entre 0,77 a 2, 51g. $\mathrm{mm} / \mathrm{m}^{2}$.dia KPa Os fatores de maior influência sobre o PVA nos filmes são as concentrações de glicerol e óleo essencial, que aumentam a permeabilidade conforme o aumento dessas variáveis. Em relação à espessura, os valores estavam na faixa de 0,02 a $0,04 \mathrm{~mm}$. Conclui-se que os filmes de colágeno obtiveram boas propriedades mecânicas, adequada aparência visual e fácil manuseio, bem como baixa PVA e baixa solubilidade em água. O óleo essencial foi eficaz na estrutura do filme, pois melhorou a aparência e facilitou o manuseio.

Palavras-chave: argila, propriedades, Syzygium aromaticum

ABSTRACT - This objective of this study was to develop and characterized films based on collagen and analyze the effects of concentrations of clay, plasticizer and essential oil on its characteristics. The solutions were prepared according to an experimental design of $2^{3}$ with 3 central points. The films were produced by the "casting" technique, which consists on surface drying, and were characterized by the color, opacity, tensile strength, solubility, water vapor permeability and thickness. Lightness values ranged between 66.76 and 96.03, the brightness decreased with increasing the concentrations of clay, oil and glycerol. The opacity values showed an increase with the addition of glycerol, ranging from 1.96 to $44.24 \%$. The values of tensile strength ranged from 5 to $14 \mathrm{MPa}$, and the solubility values, from 2.14 to $7.97 \%$. In the $5 \%$ level, solubility and tensile strength analyzes were not significant. The water vapor permeability (WVP) ranged from 0.77 to 2.51 (g.mm/KPa.m².d). The most influent factors on the permeability to water vapor in the films are the glycerol and essential oil concentrations, which increases the permeability as these variables increase. In relation to the thickness, the concentration of montmorillonite clay interfered in the range from 0.02 to $0.04 \mathrm{~mm}$, and as higher its concentration, greater the thickness. It was concluded that the collagen films obtained good mechanical properties, adequate visual appearance, easy handing and low permeability to water vapor and low water solubility. The essential oil was effective in the structure of the film, since that it improves the look and easier handling.

Keywords: argil, properties, Syzygium aromaticum

Financiamento: Os autores reportam que não houve suporte e auxílio financeiro.

Conflito de interesse: Os autores declaram que não há conflito de interesse.

Como referir esse documento (ABNT):

NAKASHIMA, A.Y.; CHEVALIER, R.C.; CORTEZ-VEGA, W.R. Desenvolvimento e caracterização de filmes de colágeno com adição de óleo essencial de cravo-da-índia. Journal of Bioenergy and Food Science, Macapá, v.3, n.1, p.50-57, jan./mar., 2016. DOI:10.18067/jbfs.v3i1.86 


\section{INTRODUÇÃO}

A incorporação de argila em filmes poliméricos diminui a solubilidade e a permeabilidade a vapor de água. ${ }^{1}$ As embalagens de alimentos exercem um papel fundamental na conservação de produtos alimentícios, porém as embalagens tradicionais necessitam de melhorias para atender à expectativa do consumidor por produtos seguros, com maior vida útil, nutritivos e mais próximos ao natural, porém com menos conservantes. $^{2}$

Atualmente, um dos problemas que acometem o meio ambiente é a poluição acarretada pelo descarte de filmes plásticos. ${ }^{3}$ Uma alternativa encontrada na área de embalagens de alimentos é o desenvolvimento de filmes obtidos a partir de biopolímeros que possam substituir os materiais sintéticos. ${ }^{4}$

Um dos processos mais utilizados no desenvolvimento de filmes biopolimérico é o "casting", que compreende o preparo de uma solução filmogênica, aplicação dessa solução num suporte adequado, seguida de secagem em condições controladas. Dessa forma, para a preparação de filmes biopolimérico a partir de novas matérias primas biológicas, é necessário obter o biopolímero e ter o conhecimento de suas propriedades, principalmente da sua solubilidade em água e sua capacidade de interação intermolecular. ${ }^{4}$ Filmes de gelatina foram preparados com sucesso pela técnica de moldagem por compressão térmica. ${ }^{5}$

As argilas têm sido bastante estudadas como material de reforço em filmes por serem abundante na natureza e de baixo custo. Os filmes com adição de argila podem ter suas propriedades mecânicas e de barreira reforçadas pela dispersão uniforme de argilas, que diminui a mobilidade molecular da matriz. ${ }^{6}$

As propriedades dos óleos essenciais, extraídos de plantas aromáticas e medicinais têm sido muito exploradas devido às suas propriedades biológicas. O uso de compostos antimicrobianos naturais tem se intensificado com a intenção de uso na conservação de alimentos, por serem mais seguros em relação aos aditivos químicos. ${ }^{7}$

As embalagens com óleos essenciais têm atraído atenção dos pesquisadores em virtude do sabor e do aroma diferenciado, das suas propriedades de conservação e atividade antimicrobiana. ${ }^{2}$

O objetivo deste trabalho foi desenvolver e caracterizar filmes à base de colágeno e estudar os efeitos das concentrações de argila, óleo essencial e glicerol sobre as propriedades mecânicas e propriedades de barreira das soluções filmogênicas

\section{MATERIAL E MÉTODOS}

O biopolímero empregado neste trabalho foi o colágeno em pó (Luchebras, Rio Grande do Sul, Brasil). O agente plastificante utilizado foi glicerol (Vetec, Química Fina), e a argila foi o Montmorilonita K10 (MMT, Sigma-Aldrich).

O óleo essencial de cravo-da-índia (Petit Marie, São Paulo, Brasil). Os demais reagentes, bem como os equipamentos utilizados, foram provenientes dos laboratórios da Faculdade de Engenharia da Universidade Federal da Grande Dourados (FAEN-UFGD).

\section{a) Produção dos filmes}

Realizou-se um planejamento fatorial fracionário $2^{3}$ ( 8 experimentos) com 3 repetições no ponto central, totalizando 11 experimentos.

Foram utilizados 2 níveis $(-1,+1)$ representando as variáveis codificadas e 3 variáveis independentes: argila montmorilonita (MMT), plastificante glicerol (G) e óleo essencial de cravo (OEC) no preparo da solução filmogênica (SF) que originou os filmes, conforme demonstrado na Tabela 1.

Tabela 1. Variáveis utilizadas no planejamento experimental para desenvolvimento de filmes de colágeno.

Table 1. Variables used in the experimental design for the development of collagen films.

\begin{tabular}{cccc}
\hline Níveis das & \multicolumn{3}{c}{ Variáveis reais } \\
\cline { 2 - 4 } Variáveis & MMT & OEC & G \\
\hline-1 & 0,2 & 0,12 & 25,0 \\
0 & 0,5 & 0,30 & 30,0 \\
+1 & 0,8 & 0,48 & 35,0 \\
\hline
\end{tabular}

MMT: Argila Montmorilonita (g/100g de solução); OEC: Óleo essencial de cravo ( $\mathrm{mL} / 100 \mathrm{~g}$ de solução) e G: Glicerol (\%/100g de colágeno).

MMT: Montmorillonite clay (g / 100g solution); OEC: Essential oil of clove (ml/100g solution) and G: Glycerol (\% / 100g collagen).

A matriz do planejamento do tipo Fatorial Fracionário com 3 variáveis e 3 pontos centrais está demonstrada na Tabela 2. 
Tabela 2. Matriz do planejamento experimental utilizada para o desenvolvimento de filmes de colágeno.

Table 2. Experimental design matrix used for the development of collagen films.

\begin{tabular}{cccc}
\hline \multirow{2}{*}{ Experimentos } & \multicolumn{3}{c}{ Variáveis reais } \\
\cline { 2 - 4 } & MMT $(\mathrm{g})$ & OEC $(\mathrm{mL})$ & $\mathrm{G}(\%)$ \\
\hline 1 & 0,2 & 0,12 & 25,0 \\
2 & 0,2 & 0,12 & 35,0 \\
3 & 0,2 & 0,48 & 25,0 \\
4 & 0,2 & 0,48 & 35,0 \\
5 & 0,8 & 0,12 & 25,0 \\
6 & 0,8 & 0,12 & 35,0 \\
7 & 0,8 & 0,48 & 25,0 \\
8 & 0,8 & 0,48 & 35,0 \\
9 & 0,5 & 0,30 & 30,0 \\
10 & 0,5 & 0,30 & 30,0 \\
11 & 0,5 & 0,30 & 30,0 \\
\hline
\end{tabular}

MMT: Argila Montmorilonita, OEC: Óleo essencial de cravo e G: Glicerol.

MMT: Montmorillonite clay; OEC: Essential oil of clove and G: Glycerol.

A solução filmogênica foi preparada hidratando-se $2 \mathrm{~g}$ de colágeno em $100 \mathrm{~mL}$ de água destilada durante 30 minutos em temperatura ambiente. Após esse período, a solução foi aquecida em placa de aquecimento sob agitação magnética. Ao atingir a temperatura de $83^{\circ} \mathrm{C}$, foram adicionados argila, plastificante, óleo essencial, por 30 minutos, nas concentrações de acordo com o planejamento experimental das Tabelas 1 e 2 .

Para a formação do filme, utilizou-se o método "casting", onde se espalhou $30 \mathrm{~mL}$ da solução filmogênica em placas de acrílico com $9 \mathrm{~cm}$ de diâmetro, e foram secados à temperatura de $40^{\circ} \mathrm{C}$ na estufa de secagem com circulação de ar (Marconi, modelo MA035, São Paulo, Brasil) até que a água fosse totalmente evaporada. Após a secagem, os filmes foram armazenados por $24 \mathrm{~h}$, até o início das análises, em dessecadores mantidos a $20^{\circ} \mathrm{C}$ e umidade relativa de $75 \%$. A umidade relativa foi controlada usando solução saturada de cloreto de sódio, até o início das análises.

\section{b) Espessura}

A espessura dos filmes foi obtida utilizandose um paquímetro digital (Digimess, São Paulo, Brasil), e determinada pela média de oito medidas aleatórias em diferentes partes do filme.

\section{c) Cor e opacidade}

A determinação da cor e da opacidade de cada filme foi realizada usando colorímetro Chroma
Meter CR 400 (Konica Minolta, Japão). Os parâmetros $L^{*}, a^{*}$ e $b^{*}$ foram determinados diretamente pelo colorímetro sobrepondo-se os filmes sobre um padrão branco e um padrão preto. Para a determinação da opacidade foi calculada a relação entre a opacidade dos filmes sobreposto ao padrão preto (Ppreto) e ao padrão branco (Pbranco), segundo a Equação 1.

Opacidade $(\%)=\frac{P_{\text {preto }}}{P_{\text {branco }}} * 100 \quad$ Eq. (1)

\section{d) Propriedades mecânicas}

As propriedades mecânicas dos filmes foram realizadas em um texturômetro (Stable Micro Systems modelo TA.XT plus), empregando-se metodologia ASTM D882-91. ${ }^{8}$ A separação inicial das garras e a velocidade do probe foram de $50 \mathrm{~mm}$ e $1 \mathrm{~mm} / \mathrm{s}$, respectivamente. As amostras foram cortadas em tiras de $7 \mathrm{~cm}$ de comprimento e $4 \mathrm{~cm}$ de largura. A resistência à tração (RT) foi calculada pela e Equação 2.

$R T=\frac{F m}{A}$

Onde:

$\mathrm{RT}=$ Resistência à tração ( $\mathrm{MPa}$ )

$\mathrm{Fm}=$ Força máxima no momento da ruptura do filme (N) $A=$ Área da secção transversal do filme $\left(\mathrm{m}^{2}\right)$

\section{e) Solubilidade em água}

Para a determinação da solubilidade, os filmes foram recortados em quadrados de $2 \mathrm{~cm}$. A matéria seca inicial foi obtida em balança analítica após secagem em estufa a $105^{\circ} \mathrm{C}$ por $24 \mathrm{~h}$. Após a primeira pesagem, os discos foram imersos em recipientes contendo $50 \mathrm{~mL}$ de água e colocados a agitação constante de 150 rpm em Shaker (Marconi, São Paulo) por 24h, a temperatura ambiente. Em seguida, as amostras foram removidas e secas em estufa (Marconi, São Paulo) a $105^{\circ} \mathrm{C}$ por $24 \mathrm{~h}$ para determinar a matéria seca que não se dissolveu em água. ${ }^{1}$

\section{f) Permeabilidade ao vapor de água (PVA)}

A taxa de permeabilidade ao vapor de água foi determinada pela norma ASTM E96. ${ }^{9} \mathrm{~A}$ amostra de filme foi selada na abertura circular de uma célula de permeação contendo cloreto de cálcio anidro ( $0 \%$ de HR). Estas células de permeação foram colocadas em dessecadores com uma solução saturada de cloreto de sódio ( $75 \%$ HR) e mantidas a 
$20^{\circ} \mathrm{C}$. O ganho de massa através do filme foi analisado a cada $24 \mathrm{~h}$, durante 7 dias. A permeabilidade ao vapor de água foi calculada pela Equação 3.

$P V A=\frac{\Delta W}{t} * \frac{X}{A \Delta P}$

Onde:

PVA - Permeabilidade ao vapor $\mathrm{H}_{2} \mathrm{O}$ (g.mm/KPa.dia. $\mathrm{m}^{2}$ )

$\Delta \mathrm{W}=$ Ganho de massa pelo dessecante $(\mathrm{g})$

$X=$ Espessura do filme $(\mathrm{mm})$

$\mathrm{t}=$ Tempo de incubação (dias)

$A=$ Área da superfície do filme exposto $\left(\mathrm{m}^{2}\right)$

$\Delta \mathrm{P}=$ Diferença de pressão parcial $(\mathrm{KPa})$

\section{g) Análise estatística}

As análises foram realizadas em triplicata e os resultados foram apresentados pela média e o desvio padrão. Os resultados obtidos foram avaliados estatisticamente através de análise de variância (ANOVA) seguida do teste de Tukey a 5\% de significância, utilizando o programa STATISTICA 6.1 .

\section{RESULTADOS E DISCUSSÃO}

Durante o preparo dos filmes, observou-se que aqueles que possuíam maior quantidade de óleo essencial foram removidos das placas de acrílico com maior facilidade do que aqueles com menor quantidade.

Retirados das placas, os filmes apresentaram tendência a se encolher e grudar. Notou-se que a adição de óleo essencial contribuiu para o manuseio, devido à menor aderência e encolhimento.

Em relação ao atributo cor, os valores de $L^{*}$, $a^{*}$ e $b^{*}$ apresentaram diferença significativa em nível de $5 \%$. A coloração nos filmes varia de acordo com a composição dos seus componentes (glicerol, argila, óleo essencial e colágeno). Os valores do parâmetro $b^{*}$ indicam que todos os filmes apresentaram coloração tendendo ao amarelo, típicas do colágeno. 0 parâmetro $a^{*}$ próximos de 0 implica em filmes com pouca tendência a cor esverdeada, pois quanto maior o valor negativo do parâmetro, mas tende ao verde.

Os resultados referentes às análises de cor e opacidade nos filmes de colágeno podem ser observados na Tabela 3.

Tabela 3. Resultados de cor e opacidade dos filmes de colágeno.

Table 3. Results of color and opacity of collagen films.

\begin{tabular}{|c|c|c|c|c|}
\hline \multirow[b]{2}{*}{ Ensaio } & \multicolumn{3}{|c|}{ Parâmetros } & \multirow{2}{*}{ Opacidade (\%) } \\
\hline & $\mathrm{L}^{*}$ & Chroma a* & Chroma b* & \\
\hline 1 & $96,03 \pm 0,42^{a}$ & $-0,52 \pm 0,04^{a}$ & $6,13 \pm 0,89^{b}$ & $12,43 \pm 0,77^{c}$ \\
\hline 2 & $95,82 \pm 0,55^{a}$ & $-0,46 \pm 0,07^{a}$ & $5,82 \pm 0,18^{b}$ & $11,96 \pm 0,48^{c}$ \\
\hline 3 & $94,35 \pm 0,17^{\mathrm{a}}$ & $-0,63 \pm 0,03 a b$ & $8,69 \pm 0,04^{a}$ & $13,60 \pm 0,84^{c}$ \\
\hline 4 & $70,38 \pm 0,38^{b}$ & $-2,32 \pm 0,14^{d}$ & $-3,62 \pm 1,07^{d}$ & $32,07 \pm 0,57^{b}$ \\
\hline 5 & $93,83 \pm 0,53^{a}$ & $-0,93 \pm 0,04^{c}$ & $11,49 \pm 1,07^{a}$ & $13,07 \pm 0,41^{c}$ \\
\hline 6 & $94,41 \pm 0,17^{a}$ & $-0,51 \pm 0,03^{a}$ & $7,71 \pm 0,13^{a}$ & $13,22 \pm 0,35^{c}$ \\
\hline 7 & $94,41 \pm 0,32^{a}$ & $-0,75 \pm 0,10^{b c}$ & $9,06 \pm 0,71^{a}$ & $13,86 \pm 0,41^{c}$ \\
\hline 8 & $66,76 \pm 3,66^{c}$ & $-2,20 \pm 0,13^{d}$ & $-2,98 \pm 0,02^{c d}$ & $33,62 \pm 1,23^{b}$ \\
\hline 9 & $69,68 \pm 0,24$ bc & $-2,31 \pm 0,02^{d}$ & $-3,17 \pm 0,22^{c d}$ & $34,23 \pm 1,58^{b}$ \\
\hline 10 & $69,54 \pm 0,32 \mathrm{bc}$ & $-2,22 \pm 0,02^{d}$ & $-1,88 \pm 0,11^{c d}$ & $42,34 \pm 2,12^{a}$ \\
\hline 11 & $69,66 \pm 0,53 \mathrm{bc}$ & $-2,17 \pm 0,05^{d}$ & $-1,76 \pm 0,30^{c d}$ & $44,24 \pm 1,71^{a}$ \\
\hline
\end{tabular}

* Média de 3 repetições \pm desvio padrão. Letras minúsculas diferentes na mesma coluna diferem entre si, pelo Teste de Tukey $(p \leq 0,05)$.

* Average of 3 replications \pm standard deviation. different lowercase letters in the same column differ by Tukey test $(p \leq 0.05)$.

A luminosidade dos filmes varia $66,76 \pm 3,66$ a $96,03 \pm 0,42$. Com o aumento da concentração de óleo essencial de cravo e glicerol utilizados nos filmes, diminui-se a luminosidade. A maioria dos filmes elaborados apresentou luminosidade acima de 90, de forma que, quanto mais próximos de 100, mais transparentes. Portanto, estes podem ser considerados claros. A partir dos resultados da Tabela 3 foram gerados a superfície de resposta para os parâmetros que foram preditivos e significativos. Na Figura 1 pode-se observar que quanto menor a concentração de óleo essência de cravo e glicerol, menor é a luminosidade. 


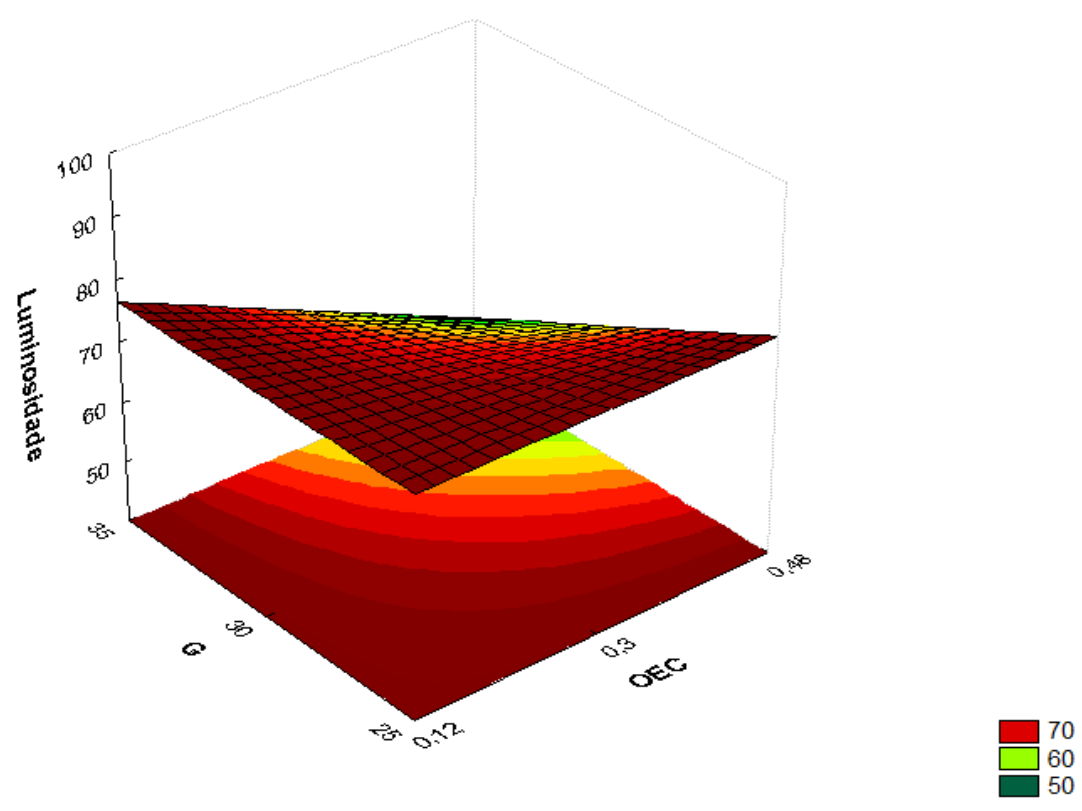

Figura 1. Superfície de resposta da luminosidade em função das concentrações de $\mathrm{G}$ (g/100g de colágeno) e OEC ( $\mathrm{mL} / 100 \mathrm{~g}$ de solução).

Figure 1. Response surface luminosity in function of concentrations of G (g/ $100 \mathrm{~g}$ of collagen) and OEC ( $\mathrm{ml} / \mathrm{100g}$ solution).

Os filmes do ponto central foram os que obtiveram maior opacidade. Valores baixos e altos na opacidade indicam filmes transparentes e opacos, respectivamente. Os filmes opacos dificultam a transmissão de luz, o que pode ser uma característica desejada dependendo do produto, ou seja, quando a luz é um fator de deterioração ou até mesmo para mascarar um produto. Davanço et al., ${ }^{10}$ encontrou valores de opacidade entre 16,8 e $24,4 \%$ em filmes compostos de gelatina, triacetina, ácido esteárico ou capróico, sendo que filmes com ácido esteárico apresentaram-se mais opacos. Silva ${ }^{11}$ desenvolveu filmes de gelatina de peles de Bijupirá e observou que a opacidade dos filmes foi maior quando utilizado maiores concentrações de proteína e de plastificante.

A opacidade dos filmes apresentou um aumento com a adição de glicerol, conforme pode ser observado na Figura 2, sendo que o ponto central da MMT (0,5 g/100g de colágeno) foi o que apresentou maior opacidade.
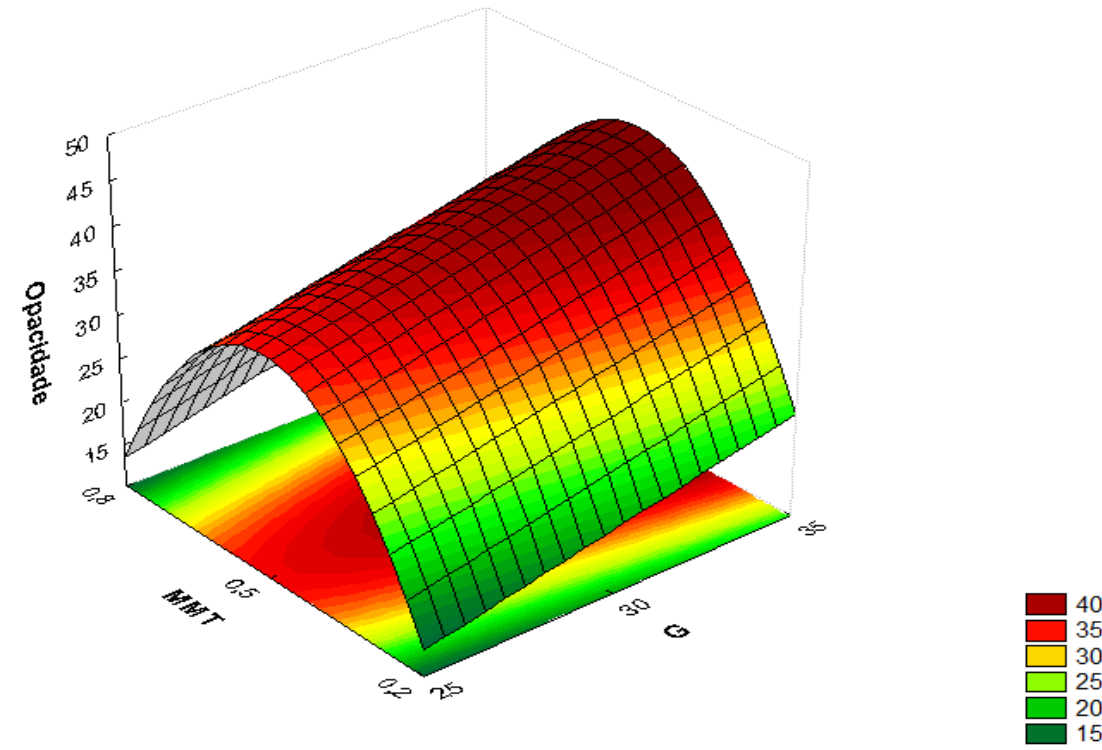

Figura 2. Superfície de resposta da opacidade em função das concentrações de glicerol (g/100g de colágeno) e MMT (g/100g de solução).

Figure 2. Response surface opacity in function of concentrations of glycerol $(\mathrm{g} / 100 \mathrm{~g}$ collagen) and MMT (g / $100 \mathrm{~g}$ solution) 
A espessura dos filmes variou de $0,02 \mathrm{~mm}$ a $0,04 \mathrm{~mm}$, mesmo havendo uma padronização de 30 gramas de solução filmogênica nas placas de petri, conforme os dados apresentados na Tabela 1. 0 aumento da argila montmorilonita provocou aumento na espessura. Os valores de espessura estão próximos ao encontrado por Matta Junior et al., ${ }^{12}$ para filmes de amido de ervilha que variou entre $0,055 \mathrm{~mm}$ para o tratamento com apenas amido $3 \%$ até $0,098 \mathrm{~mm}$ para o tratamento que continha $5 \%$ de amido e $20 \%$ de glicerol, segundo o autor a espessura foi maior quanto mais elevado o teor de amido e de glicerol na solução filmogênica e próximos ao encontrado por Fakhouri et al., ${ }^{13} \mathrm{com}$ filmes a base de amido e gelatina que variou entre $0,053 \mathrm{~mm}$ a $0,063 \mathrm{~mm}$.

Segundo Sobral ${ }^{14}$ a força na perfuração, a permeabilidade ao vapor de água, a diferença de cor e a opacidade dos filmes aumentam linearmente com a espessura dos corpos de prova.

Os resultados referentes à análise de espessura, solubilidade e permeabilidade podem ser observados na Tabela 4.

Tabela 4. Resultados obtidos de espessura, solubilidade e permeabilidade.

Table 4. Results obtained thickness, solubility, and permeability.

\begin{tabular}{|c|c|c|c|c|}
\hline \multirow[b]{2}{*}{ Ensaio } & \multicolumn{4}{|c|}{ Parâmetros } \\
\hline & Espessura (mm) & Solubilidade (\%) & $\begin{array}{c}\text { Permeabilidade } \\
\text { (g.mm/m².dia.KPa) }\end{array}$ & $\begin{array}{l}\text { Resistência a tração } \\
\text { (MPa) }\end{array}$ \\
\hline 1 & $0,02 \pm 0,01^{a}$ & $4,40 \pm 0,01^{h}$ & $1,17 \pm 0,03^{b c}$ & $12,3 \pm 0,4^{b}$ \\
\hline 2 & $0,02 \pm 0,01^{a}$ & $7,97 \pm 0,02^{b}$ & $2,00 \pm 0,10^{b}$ & $7,0 \pm 0,1^{\mathrm{e}}$ \\
\hline 3 & $0,02 \pm 0,01^{a}$ & $5,64 \pm 0,02^{e}$ & $2,11 \pm 0,09^{b}$ & $9,5 \pm 0,2^{c}$ \\
\hline 4 & $0,02 \pm 0,01^{a}$ & $7,21 \pm 0,01^{d}$ & $2,09 \pm 0,06^{b}$ & $10,1 \pm 0,1^{c}$ \\
\hline 5 & $0,03 \pm 0,02^{a}$ & $7,52 \pm 0,05^{c}$ & $2,51 \pm 0,13^{b}$ & $8,0 \pm 0,1^{d}$ \\
\hline 6 & $0,03 \pm 0,01^{a}$ & $2,14 \pm 0,00^{k}$ & $2,04 \pm 0,13^{b}$ & $12,4 \pm 0,5^{b}$ \\
\hline 7 & $0,02 \pm 0,01^{a}$ & $4,96 \pm 0,06^{\mathrm{g}}$ & $2,30 \pm 0,29^{b}$ & $10,2 \pm 0,2^{c}$ \\
\hline 8 & $0,04 \pm 0,01^{a}$ & $11,17 \pm 0,07^{a}$ & $4,99 \pm 1,62^{a}$ & $5,1 \pm 0,1^{f}$ \\
\hline 9 & $0,03 \pm 0,01^{a}$ & $3,89 \pm 0,02^{i}$ & $0,79 \pm 0,20^{c}$ & $7,1 \pm 0,3$ de \\
\hline 10 & $0,03 \pm 0,01^{a}$ & $5,12 \pm 0,01^{f}$ & $1,97 \pm 0,18^{b}$ & $9,4 \pm 0,3^{c}$ \\
\hline 11 & $0,03 \pm 0,01^{a}$ & $3,27 \pm 0,02^{j}$ & $0,77 \pm 0,04^{c}$ & $14,2 \pm 0,6^{a}$ \\
\hline
\end{tabular}

* Média de 3 repetições \pm desvio padrão. Letras minúsculas diferentes na mesma coluna diferem entre si, pelo Teste de Tukey $(p \leq 0,05)$.

* Average of 3 replications \pm standard deviation. different lowercase letters in the same column differ by Tukey test $(p \leq 0.05)$.

A permeabilidade ao vapor de água variou de

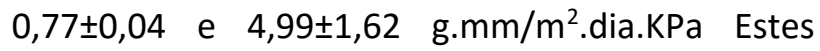
valores são um pouco abaixo dos valores encontrado por Davanço et al. ${ }^{10}$ com filmes compostos de gelatina, triacetina, ácido esteárico ou capróico $\left(1,9 \pm 0,4\right.$ e $8,3 \pm 0,4$ g.mm/ $/ \mathrm{m}^{2}$.dia.KPa) e Fakhouri et al., ${ }^{13}$ com filmes a base de amido e gelatina onde a permeabilidade ao vapor de água variou de 4,22 a $5,53 \mathrm{~g} \cdot \mathrm{mm} / \mathrm{m}^{2}$.dia. $\mathrm{kPa}$ para os filmes.

Através dos resultados da Tabela 4 observase que com o aumento do plastificante e do óleo essencial ocorre um aumento da permeabilidade. Segundo Nagarajan et al., ${ }^{15}$ a adição de plastificante apresenta caráter fortemente hidrofílico. Batista et al., ${ }^{16}$ atribuíram um aumento na permeabilidade ao vapor de água em filmes a medida que aumentava o teor de ácidos graxos.

Neste estudo a solubilidade dos filmes de colágeno variou de $2,14 \%$ a $7,97 \%$, conforme demonstrado na Tabela 4. Resultados diferentes foram encontrados por Ferreira ${ }^{17}$ em estudos feitos com filmes a partir de proteína de pescado e argilas 
incorporados com óleos essenciais, onde a solubilidade obtida variou de $22,32 \%$ a $33,54 \%$.

Em relação aos parâmetros de solubilidade pode-se observar que todos os ensaios apresentaram diferença significativa entre si ( $p>0,05)$. Para os testes de permeabilidade o ensaio 8 apresentou o maior valor (4,99 g.mm/ $/ \mathrm{m}^{2}$.dia. $\left.\mathrm{KPa}\right)$. A resistência à tração dos filmes analisados foi maior no ensaio 11 sendo que este diferiu significativamente dos demais ensaios analisados. A resistência à tração dos filmes depende das interações entre as moléculas, ou seja, da formação de ligações moleculares fortes ou numerosas entre as cadeias.

Alexandre e Dubois, ${ }^{6}$ afirma que filmes podem ter reforço em suas propriedades mecânicas com adição de argila, porém, no estudo feito com filmes de colágeno, não ocorreu diferença significativa com a adição da argila, ou seja, não houve reforço em suas propriedades mecânicas, isso pode ter ocorrido devido às baixas concentrações utilizadas no estudo.

A resistência à tração variou entre 5,1 e 14,2 $\mathrm{MPa}$. Resultados diferentes foram encontrados por Fakhouri et al., ${ }^{13}$ que trabalharam com filmes de gelatina, adicionado de ácido láurico, variando entre 31,83 a 57,38 MPa, onde se notou que a resistência diminuía com a concentração de lipídios. CortezVega et al., ${ }^{18}$ também encontraram resultados diferentes em seu estudo com filmes biopoliméricos obtidos de isolado proteico de corvina e argila montmorilonita, variando entre 7,2 e 7,3 $\mathrm{MPa}$.

\section{CONCLUSÃO}

Podemos concluir que o óleo essencial foi eficaz na estrutura dos filmes, pois melhorou a aparência e facilitou o manuseio. Os filmes de colágeno apresentaram baixa solubilidade. $\mathrm{Na}$ espessura do filme, houve um aumento conforme a concentração da argila montmorilonita. Quando aumentadas as concentrações de OEC e glicerol, existe um aumento da permeabilidade do filme de colágeno e aumento da luminosidade, o que de fato é de extrema importância uma vez que filmes com alta luminosidade se assemelhariam a filmes plásticos, muito utilizados para embalagens de alimentos.

\section{AGRADECIMENTOS}

Ao Laboratório de Bioengenharia da FAENUFGD da Universidade Federal da Grande Dourados pela disponibilização de materiais equipamentos.

\section{CONTRIBUIÇÃO DOS AUTORES}

1 - Condução e avaliação do experimento, análises estatísticas e elaboração do artigo:

William Renzo Cortez-Vega

Ailim Yuki Nakashima

Raquel Costa Chevalier 9

2 - Planejamento, orientação e revisão final do artigo:

William Renzo Cortez-Vega

\section{REFERÊNCIAS}

1. WILLIAM, R.C.V., DANIELA, C. B., SANDRIANE, P. and CARLOS, P. Preparation and characterization of nanocomposite film from Whitemouth croaker (Micropogonias furnieri) protein isolate modified with montmorillonite. International Food Research Journal, v.22, n.3, p.1053-1058, 2015.

2. SOARES, N.F.F.; SILVA, S.A.; PIRES, A.C.S.; CAMILLOTO, G.P.; SILVA, P.S. Novos desenvolvimentos e aplicações em embalagens de alimentos. Revista Ceres, v.56, p.370-378, 2009.

3. Silva, W. A.; PeReIRA, J; CARVAlho C. W. P.; FERRUA, F. Q. Determinação da cor, imagem superficial topográfica e ângulo de contato de biofilmes de diferentes fontes de amido. Ciência e Agrotecnologia, v.31, n.1, p.154-163, 2007.

4. MONTERREY-QUINTERO, E. S.; SOBRAL, P.J.A. Preparo e caracterização de proteínas miofibrilares de tilápia-do-nilo para elaboração de biofilmes. Pesquisa Agropecuária Brasileira, Brasília, v.35, n.1, p.179-189, 2000.

5. CHUAYNUKUL, K.; PRODPRAN, T.; BENJAKUL, S. Properties of thermo-compression molded bovine and fish gelatina films as influenced by resin preparation condition. International Food Research Journal, v.22, n.3, p.1095-1102, 2015.

6. ALEXANDRE, M.; DUBOIS, P. Polymer-layered silicate nanocomposites: preparation, properties and uses of a new class of materials. Materials Science and Engineering, v.28, p.1-63, 2000.

7. SANTOS, S.C.; FERREIRA, F.S.; DAMIÃO, A.O.; BARROS, T.F.; ROSS-ALVA, J.C.; FERNANDEZ, L.G. Avaliação da atividade antibacteriana dos extratos de Avicennia schaueriana Stapt \& Leechm. ex Moldenke, Verbenaceae. Revista Brasileira de Farmacognosia, v.20, p.124-129, 2010. 
8. ASTM. AMERICAN SOCIETY FOR TESTING AND MATERIALS. Standard test methods for tensile properties on thin plastic sheeting. Método: D00882-00. In: ASTM annual book of ASTM standarts, p.160-168, 2000.

9. ASTM. AMERICAN SOCIETY FOR TESTING AND MATERIALS. Standard methods of water vapor transmission of materials. Método: E00996-00. In: ASTM annual book of ASTM standards, p. 907-914, 2000.

10. DAVANÇO, T.; TANADA-PALMU, P.; GROSSO, C. Filmes compostos de gelatina, triacetina, ácido esteárico ou capróico: efeito do pH e da adição de surfactantes sobre a funcionalidade dos filmes. Ciência e Tecnologia de Alimentos, v.27, n.2, p.408416, 2007.

11. SILVA, R.S.G. Obtenção de peles de bijupirá (Rachycentron canadum), modificação e produção de filmes. Tese de Doutorado em Engenharia e Ciência de alimentos - Universidade Federal do Rio Grande, 169f, Rio Grande- RS, 2013.

12. MATTA JR, M.D.; SARMENTO, S.B.S.; SARANTOPOULOS, C.I.G. L.; ZOCCHI, S.S. Propriedades de barreira e solubilidade de filmes de amido de ervilha associado com goma xantana e glicerol. Polímeros, v.21, n.1, p.67-72, 2011.

13. FAKHOURI, F.M.; FONTES, L.C.B.; GONÇALVES, P.V.D.M.; MILANEZ, C.R., STEEL, C.J., COLLARESQUEIROZ, F.P. Filmes e coberturas comestíveis compostas à base de amidos nativos e gelatina na conservação e aceitação sensorial de uvas Crimson.
Ciência e Tecnologia de Alimentos. v.27, p.369375, 2007.

14. SOBRAL, P.J. A Influência da espessura de biofilmes feitos à base de proteínas miofibrilares sobre suas propriedades funcionais.Pesquisa Agropecuária Brasileira, v.35, n. 6, p. 1251-1259, 2000.

15. NAGARAJAN, M.; BENJAKUL, S.; PRODPRAN, T.; SONGTIPYA, P. Properties of film from splendid squid skin gelatin with various extraction temperatures. Food Hydrocolloids, v.29, p.489496, 2012.

16. BATISTA, J.A.; TANADA-PALMU, P.S.; GROSSO, C. R.F. Efeito da adição de ácidos graxos em filmes à base de pectina. Ciência e Tecnologia de Alimentos, v.25, n.4, p.781-788, 2005.

17. FERREIRA, F. A. Filmes nanocompósitos antimicrobianos obtidos a partir de proteínas de pescado e nanoargila incorporados com óleos essenciais. Tese de Doutorado em Engenharia e Ciência de Alimentos- Escola de Química, Universidade Federal do Rio Grande, 105f, Rio Grande -RS, 2014

18. CORTEZ-VEGA, W.R.; BAGATINI D.C.; SOUZA, J.T.A. Biofilmes nanocompósitos obtidos de isolado proteico de corvina (Micropogonias furnieri) e Montmorilonita: avaliação das propriedades físicas, mecânicas e de barreira. Brazilian Journal of food Technology, v.16, n.2, p.90-98, 2013. 